\title{
Die Textgliederung in Christian Pudors Grammatik (1672)
}

\section{Text Structure in Christian Pudor's Grammar Book (1672)}

\author{
Jana Adamusová, Karsten Rinas
}

\begin{abstract}
Christian Pudor's grammar book has not received a lot of attention, despite its importance as the first German grammar book, which gives a syntactically based theory on punctuation. Our paper focuses on another aspect of his book, that of Pudor's very specific and progressive way of visualizing of the text structure. Nowadays, it is typical to use a linear system of numbering. Pudor used numbering in combination with a system of brackets on the page margin, which often cross multiple pages. These brackets are supposed to visualize the logical structure of the text, and its layers and substructures. We can compare this two-dimensional system with a similar attempt by Gottlob Frege in the field of Logic. Frege developed a notational system for logical formulas which is two-dimensional in space, unlike the contemporary linear way, which originates in the notation of Peano and Russell.

Although the usage of semiotic possibilities for text structuring did not develop chronologically, and the structural elements are used at different times with various intensities, it is obvious, that the linear approach was more successful. It is worth discussing why this is so and whether we can be inspired by those more complex structural systems now that there are better technical possibilities.
\end{abstract}

\section{Keywords}

Christian Pudor; grammar book; text structure; two-dimensionality; brackets; Gottlob Frege

Diese Studie ist entstanden im Rahmen des von der Philosophischen Fakultät der Palacký-Universität Olomouc geförderten Projekts "Hermeneutische Linguistik" " Hermeneutická lingvistika” (IGA_FF_2016_020). 


\section{Einleitung}

Christian Pudors Werk Der Teutschen Sprache Grundrichtigkeit und Zierlichkeit, das im Jahre 1672 erschienen ist, hat nicht so große Aufmerksamkeit erregt, wie andere zeitgenössische Grammatiken. Nichtsdestoweniger handelt es sich um ein beachtenswertes und progressives Werk. Das kleine Buch enthält als die erste deutsche Grammatik eine Satzlehre sowie die erste Interpunktionslehre, die eine klare syntaktische Grundlage aufweist (vgl. 3.1).

Wir wollen uns im Folgenden jedoch auf einen anderen Aspekt dieses Werkes konzentrieren, und zwar auf die Weise, wie Christian Pudor seinen Text graphisch strukturiert. Er benutzt nicht nur die Nummerierung, wie heute so Usus ist, sondern er verzeichnet die Beziehungen zwischen Textpassagen mithilfe von - oft sehr ausgeweiteten, sogar seitenübergreifenden - geschweiften Klammern. Das ist ein Fortschritt gegenüber dem heute üblichen Linearsystem in dem Sinne, dass die vertikale Dimension stärker ausgenutzt wird. Eine Parallele kann man in der Logik finden. Hier versucht Gottlob Frege eine zweidimensionale Notation in dieser Disziplin zu etablieren. In beiden Fällen waren die Versuche allerdings nicht von Erfolg gekrönt, sondern es haben sich eindimensionale Systeme durchgesetzt.

In folgendem Artikel stellen wir am Anfang kurz den Kontext der Entwicklung von Textgestaltung vor und danach beschäftigen wir uns hauptsächlich mit Pudors Grammatik. Nach einer knappen, den Autor betreffenden, Einführung vergleichen wir die Textgliederung von Pudor mit der von seinen Zeitgenossen und wir stellen seine eigentümliche Methode vor. Auch Freges Konzept der Notation in Logik wird angesprochen. Am Ende möchten wir kurz über die Gründe diskutieren, warum sich der progressive zweidimensionale Ansatz nicht verbreitet hat.

\section{Entwicklung der Textgestaltung}

Im Zusammenhang mit der Textgestaltung sind für unser Thema zwei wichtige Aspekte zu berücksichtigen: i) Ein geschriebener Text bietet im Gegensatz zu einem gesprochenem die Möglichkeit, die Dimensionen der Fläche zur besseren Textgestaltung auszunutzen. ii) Dies führte schon in der Antike zum partiellen Einsatz progressiver Mittel der Textgestaltung, doch wurden diese erst seit der Scholastik konsequent und systematisch genutzt.

Dies wurde bereits von Raible (1991) herausgearbeitet. Er betont, dass die gesprochene Sprache ,eindimensional' ist, obwohl uns das nicht unbedingt gleich auf den ersten Blick auffallen muss. Die Rede spielt sich linear ab, ein Laut folgt auf den anderen und wir können sie auch nur so aufeinanderfolgend rezipieren. Es ist nicht möglich, in der Rede nach vorne oder zurück zu springen.

Der geschriebene Text erstreckt sich demgegenüber auf einer Fläche, und dadurch ist er zweidimensional. Das bringt ein großes Potential mit. Die Zweidimensionalität kann zur besseren Textgestaltung und dadurch zur besseren Orientierung im Text genutzt werden. Abschnitte, Überschriften, Einrückungen, verschiedene Farben oder Buchstabengrößen, das alles hilft, sich in einem Text schneller zurechtzufinden und ihn dadurch 
leichter zu verstehen. Als Autor kann man mit dem Text flexibler arbeiten, die Textteile können hervorgehoben werden, oder es kann auf Textabschnitte verwiesen werden. Als Leser kann man bestimmte Teile des Textes schneller finden, selektiv lesen und in dem Text nach Bedarf springen. Das alles ist bei der gesprochenen Rede nicht möglich.

Die Mittel, die man für diese Arbeit mit dem Text ausnützen kann, haben sich nicht kontinuierlich entwickelt und auch ihre Benutzung war in verschiedenen Zeiten unterschiedlich.

Raible (1991: 6-7) führt als Beispiel alte juristische Texte an. Schon in dem Stadtgesetz von Málaga aus dem 1. Jahrhundert kann man eine sehr fortgeschrittene Ausnutzung von Textgliederungsmitteln beobachten. ${ }^{1}$ Die leicht zu lesende Capitalis ist durch Interpunktion in Wörter getrennt und zudem in Absätze gegliedert. Diese Absätze fangen mit einer größeren Initiale an und sind durch den hängenden Einzug gekennzeichnet und nummeriert. Die Überschriften sind eingezogen und noch zusätzlich mit dem Zeichen für Rubrizierung versehen.

Als Gegenbeispiel kann ein Brief aus dem 4. Jahrhundert dienen. ${ }^{2}$ Der in der jüngeren römischen Kursive geschriebene Text ist schwierig zu entziffern. Es handelt sich um den Gebrauch von scripta continua, d. h. die Wörter werden nicht getrennt. Nur die Anrede ist durch Worttrennung und Einzug hervorgehoben.

Mithilfe von diesen zwei Beispielen können wir klar sehen, dass die Entwicklung nicht graduell war. Höher entwickelte Mittel der Textgestaltung hat man zwar schon recht früh gekannt, trotzdem wurden sie auch in späteren Texten nicht immer ausgenutzt. In größerem Maße haben sich die Mittel erst einige Jahrhunderte später wirklich durchgesetzt.

Der Grund für die sorgfältige Ausarbeitung des Textes aus dem 1. Jahrhundert ist naheliegend. Es handelt sich um ein Stadtgesetz, d. h. er sollte von allen Bürgern gelesen, verstanden und befolgt werden. Es lag somit im Interesse des Gesetzgebers, den Text so verständlich wie möglich zu gestalten.

Raible (1991: 8) betont, dass die Entwicklung außerhalb der juristischen Sphäre deutlich langsamer war - noch bis ins 7. Jahrhundert wurde scripta continua benutzt, in der Regel ohne jegliche Interpunktion. Eine der Ausnahmen stellt die Vulgata dar, die Bibelübersetzung von Hieronymus aus dem 4. Jahrhundert. Hier wurde die Textgliederung per cola et commata benutzt, d. h. eine Schreibweise, wo Wortgruppen nach rhetorischen Kriterien durch Zeilenabsetzungen abgegrenzt werden.

Hingegen wurden Absätze erst in Scholastik in größerem Umfang markiert. Die Texte dieser Zeit waren sogar deutlich differenzierter gegliedert als viele frühe Drucktexte der Frühen Neuzeit. Erst allmählich wurde auch im Buchdruck ein hohes typographisches Niveau erreicht.

Im Laufe der Schriftgeschichte wurde auch mit Mitteln experimentiert, von denen einige wieder verschwanden. So entstanden schon in der Antike Zeichen zur initialen Markierung eines Absatzes, sog. Alinea (I). Sie fanden auch im früheren Buchdruck Verwendung.

$1 \quad$ Vgl. Abbildung 1.

2 Vgl. Abbildung 2. 
Innovative Textgliederungsmittel, die sich nicht durchgesetzt haben, finden wir nun gerade auch im Werk von Pudor.

\section{Christian Pudor und seine Grammatik}

Über den Theologen und Philologen Christian Pudor haben wir nicht viele Informationen. Er lebte von 1635 bis 1674 im heutigen Brandenburg. Er ist in Guben geboren, zwischen Jahren 1649 und 1656 studierte er an der Universität in Frankfurt (Oder), später wirkte er als Kantor und Diakon in Strausberg und als Kantor und Konrektor in Fürstenwalde/Spree. ${ }^{3}$ Im Jahre 1672 ist sein Werk unter dem verkürzten Titel Der Teutschen Sprache Grundrichtigkeit und Zierlichkeit erschienen.

\subsection{Der Teutschen Sprache Grundrichtigkeit und Zierlichkeit}

Pudors kombinierte Grammatik und Rhetorik/Stilistik ist ein knappes Werk von 143 Seiten. Der erste grammatische Teil führt in aufsteigender Reihenfolge von Buchstaben über Silben, Wörter, Redensarten (Phrases), Spruchreden (Sententiae) und Schlussreden (Periodi) bis zur vollkommenen Rede (Oratio). Pudor beschäftigt sich also mit Orthographie und Aussprache, gibt eine Wortartenlehre, beschreibt und klassifiziert verschiedene Wortverbindungen (Phrases). Seine Sententiae (Spruchreden/Sprüche) entsprechen der heutigen Einheit ,Satz', seine Periodi (Schlussreden) sind Satzverbindungen und Satzgefüge. Das letzte Kapitel, das die vollkommene Rede behandelt, bildet gleichzeitig den Übergang zu dem zweiten Teil des Buches, „Zierlichkeit“, dem stilistischen oder rhetorischen Teil. Dort betont Pudor gleich am Anfang, dass wer schön Deutsch sprechen möchte, sich der deutschen Wörter bedienen und das fremde in der Sprache möglichst vermeiden müsse. In den folgenden fünf Abschnitten bespricht Pudor die Versetzung (Transpositio), also die geänderte Wortstellung; die Verwechselung (Variatio), also die Umschreibung; des Weiteren die Erweiterung (Amplificatio); die Zusammenziehung (Contractio) sowie die Metrik.

Pudor ist nicht nur in seinem systematischen Stufenbau von Spracheinheiten originell. Seine Grammatik bringt zudem als erste eine Satzlehre. ${ }^{4}$ Vor allem die Abschnitte über Phrases, Sententiae und Periodi sind aus der syntaktischen Sicht sehr interessant. Auf Grund von seinen Erkenntnissen legt er sogar Regeln für Interpunktion fest. Es handelt sich damit ebenfalls um die erste deutsche Interpunktionstheorie. ${ }^{5}$ Die hat sich aber wahrscheinlich nicht weit verbreitet. Die etwas später erfasste Interpunktionslehre im Werk von Christian Weise aus dem Jahr 1691 hat mehr Resonanz gefunden. ${ }^{6}$

3 Vgl. Moulin-Fankhänel (1997: 234).

4 Vgl. Jellinek (1913: 186).

5 Vgl. Rinas (2014a).

6 Vgl. Rinas (2014b). 


\subsection{Textgliederung bei Pudors Zeitgenossen}

Bevor wir die Gliederung im Pudors Buch näher anschauen, wollen wir die Situation bei anderen zeitgenössischen Grammatiken skizzieren.

In seiner Hauptgliederung geht Pudor wie auch viele andere von Philipp Melanchthon und seiner Grammatica Latina aus. ${ }^{7}$ Melanchthon (1854[1526]: Sp. 245) gliedert Grammatik in vier Teile: Orthographia, Prosodia, Etymologia und Syntaxis. Orthographia soll die richtige Schreibweise lehren, Prosodia die Akzente, Etymologia ist nach Melanchthon eine Art von Flexionslehre, und Syntaxis beschäftigt sich damit, wie die Wörter miteinander in Konstruktionen und Sätzen verbunden werden können.

Solche Gliederungen wurden auch durch graphischen Mittel verdeutlicht, nicht nur bei Pudor, sondern auch bei seinen Zeitgenossen. Hier einige Beispiele:

Im Werk Deutscher Sprachlehre Entwurf (1641) von Christian Gueintz findet man größere eingerückte Überschriften, kleinere Überschriften am Rand, Ausrückungen von Text, verschiedene Schriftgröße, Initialen.

Im Buch Ausführliche Arbeit Von der Teutschen Haubt Sprache (1663) von Justus Georg Schottelius, kann man am Anfang des Teiles immer ein nummeriertes lateinisches Summarium und dementsprechend nummerierte Kapitel beobachten. Außerdem finden wir in Schottelius‘ Buch gleichfalls verschiedene Schriftgrößen, Ausrückungen von Text und Überschriften, Initialen.

Veränderung von Schriftgröße, Einrückung und Nummerierung werden auch im Werk Grund-Sätze Der Deutschen Sprachen im Reden und Schreiben (1690) von Johann Bödiker genutzt.

Ähnliche Mittel finden wir auch bei Kaspar Stieler, in seinem Werk Kurze Lehrschrift Von der Hochteutschen Sprachkunst (1691). Bei Stieler gibt es viele Tabellen, die die Textverteilung im Raum nutzen. Es gibt hier jedoch keine Linien oder Klammern.

Eine einfache Nummerierung und die oben genannten üblichen Mittel finden wir auch bei Christian Weise in seinen Curiösen Gedancken von Deutschen Brieffen (1691). Am Anfang dieses Werkes befindet sich eine ausführliche Inhaltsangabe. An einigen Stellen wird der Text in nummerierte eingerückte Listen geordnet.

\subsection{Die Gliederung von Pudors Grammatik}

Bei Pudor zeigt sich ein deutlich anderer Gebrauch von Gliederungsmitteln. Während uns in den oben genannten Büchern vorwiegend arabische bzw. römische Zahlen begegnen, nutzt Pudor diverse weitere Mittel. Neben den arabischen (1 - 14) und römischen Zahlen (I - VII) benutzt er auch Buchstaben, und zwar nicht nur die lateinischen Groß$(A-E)$ und Kleinbuchstaben $(a, b)$, sondern auch griechische $(\alpha-\delta)$ und hebräische Buchstaben $(x-\lambda)$. Allein dies lässt erahnen, wie komplex Pudors Gliederung ist. Grundsätzlich verfährt er hierbei so, dass die Hauptteile des Buches (die Grundrichtigkeit und

Vgl. Jellinek (1914: 1-3). 
die Zierlichkeit) mit den hebräischen Buchstaben, die Kapitel dann mit den römischen Buchstaben gekennzeichnet sind. Die Kennzeichnung der Unterkapitel ist verschieden.

In Pudors Werk findet sich jedoch nicht bloß eine Einteilung in Kapitel und Unterkapitel. Pudor nummeriert vielmehr auch alle Bemerkungen und Unterbemerkungen. ${ }^{8}$ In dieser geradezu pedantischen Ausgestaltung erinnert Pudors Gliederung an Wittgensteins Tractatus Logico-Philosophicus (1922: 30), ${ }^{9}$ vgl. etwa:

$1 *$ Die Welt ist alles, was der Fall ist.

1.1 Die Welt ist die Gesamtheit der Tatsachen, nicht der Dinge.

1.11 Die Welt ist durch die Tatsachen bestimmt und dadurch, dass es alle Tatsachen sind.

1.12 Denn, die Gesamtheit der Tatsachen bestimmt, was der Fall ist und auch, was alles nicht der Fall ist.

1.13 Die Tatsachen im logischen Raum sind die Welt.

1.2 Die Welt zerfällt in Tatsachen.

1.21 Eines kann der Fall sein oder nicht der Fall sein und alles übrige gleich bleiben.

Ludwig Wittgenstein nummeriert jeden Satz, aber die Untergliederung ist in einer anderen Weise durchgeführt. Die Nummern werden bei Wittgenstein immer durch eine Ziffer erweitert, um den Zusammenhang zwischen den Sätzen und ihre Abhängigkeit zu illustrieren. Ein ähnliches System ist gleichfalls von den modernen wissenschaftlichen Arbeiten bekannt. ${ }^{10}$ Nur werden da nicht einzelne Sätze, sondern Kapitel und Unterkapitel nummeriert. Die Ziffern werden meistens durch Punkte getrennt, oder es werden auch Buchstaben integriert. Auf jeden Fall herrscht jedoch eine lineare Erweiterung von den Ziffern vor, gerade wie es Wittgenstein benutzt.

Pudor benutzt aber auch andere Gliederungsmittel. Neben dem alpha-nummerischen System ändert er die Schriftgröße, oder er benutzt Kapitalschrift bei den Überschriften, was freilich auch bei anderen Autoren üblich war. Innovativ sind jedoch seine geschweiften Klammern mit Einrückungen. Geschweifte Klammern hat Pudor natürlich nicht erfunden, er benutzt sie aber in einer eigenwilligen Weise, nämlich um den Zusammenhang und die Abhängigkeit von den Bemerkungen zu zeigen. Hier sehen wir gerade den Unterschied zu Ludwig Wittgenstein, der für denselben Zweck eine andere Methode benutzt. Wir könnten sagen, dass sich Wittgenstein vorwiegend nur einer Dimension bedient und den Nachdruck auf die Linearität setzt, während Pudor stärker mit den beiden Dimensionen der schriftlichen Ebene arbeitet.

Eine klare Vorstellung von Pudors Benutzung von den geschweiften Klammern gewinnt man rasch, wenn man das Inhaltverzeichnis am Ende des Buches ${ }^{11}$ oder gleich die ersten drei Seiten der Auslegung ${ }^{12}$ ansieht.

8 Manchmal sind die Bemerkungen zu einer bloßen Liste reduziert (vgl. etwa S. 137).

9 Das Sternchen in der Zitation weist auf die Erklärung der Nummerierung, die hier nicht zitiert wird.

10 Vgl. etwa Brink (2007: 142f.).

11 Vgl. Abbildung 3.

12 Vgl. Abbildung 4. 
Die geschweiften Klammern werden für bessere Orientation mit Kleinbuchstaben am unteren Rand der Seite und am oberen Rand der nächsten Seite bezeichnet. Die Skala von a bis e zeigt wieder, dass die geschweiften Klammern, die sich manchmal durch mehrere Seiten ziehen ${ }^{13}$, ohne diese Kennzeichnung einfach zu nichtssagenden Linien am Rand würden. Die Klammern kommen nur bei den arabischen Zahlen vor, hier jedoch - bis auf eine Ausnahme ${ }^{14}$ - immer. Sie enden immer mit der Nummer, sie schließen also den dazu gehörenden Text oft nicht vollständig ein. In der Regel werden sie durch Einrückung unterstützt, d. h. die Untergliederung wird in den meisten Fällen weiter nach rechts eingerückt. Das ist natürlich in den Fällen unvermeidlich, wo links die erste Klammer noch weiter geht. Diese Vorgehensweise wird jedoch auch an übrigen Stellen durchgehalten, wo die erste Klammer schon endet (wie schon oben erwähnt wurde; die Klammer endet nämlich immer mit der Nummer, sie bezieht die noch dazugehörende Textpassage nicht mehr ein). Es gibt hier nur wenige Ausnahmen. ${ }^{15}$ Interessant ist die Stelle auf Seite 102. Im Kapitel über die Zusammenziehung in der letzten Silbe beim Buchstaben E werden 3 Varianten angeführt: inmitten der Zeile, am Ende des Verses und am Ende der Strophe (hier gibt es auch einen Fehler in Nummerierung: 1, 1, 3 anstatt 1, 2, 3). Es wird die Elision von E behandelt. Darunter stehen 2 Punkte, die erklären, in welchen Fällen E nicht elidiert wird. Man kann also diese 2 Punkte nicht den vorigen Bemerkungen zuordnen oder unterordnen, obwohl sie inhaltlich zusammenhängen - es wird in diesem Fall gerade nämlich nicht über die Elision gesprochen. Dies ist eine der Stellen im Pudors Werk, wo man sehen kann, dass sein System zwar ausgearbeitet ist, jedoch mitunter auch problematische Gliederungen enthält.

Die geschweiften Klammern werden an einigen Stellen auch auf eine andere Weise benutzt, und zwar auf die übliche, wo zu mehreren Einheiten mithilfe von der Klammer eine gemeinsame Anmerkung beigeschlossen wird. ${ }^{16}$

Als letztes Gliederungsmittel sind noch die Tabellen zu erwähnen, die eigentlich eher dadurch interessant sind, dass sie im Vergleich zu der restlichen Gliederung nicht besonders durchgearbeitet sind. Von dem Lay-out her können wir dies eine Tabelle nennen, oft fehlen jedoch jegliche gliedernde Linien. Das steht im starken Kontrast zu den überall präsenten Klammern. ${ }^{17}$

An einigen Stellen des Werkes haben wir Fehler gefunden. Typisch ist die falsche Zahlenreihenfolge, die etwa an 15 Stellen vorkommt. Dank den geschweiften Klammern sind aber diese Stellen leicht zu entziffern. Interessant ist, dass obwohl das Setzen von den geschweiften Klammern für den Typographen schwierig sein musste, es diesbezüglich

\footnotetext{
13 Vgl. Pudor (1672: 17f).

14 Vgl. Pudor (1672: 44).

15 Vgl. Pudor (1672: 8): Ganz oben sieht man eine Einteilung in kleine und große Buchstaben und gleich darunter noch eine zweiteilige Bemerkung über den Gebrauch von diesen Großbuchstaben, die eigentlich weiter nach rechts verschoben werden sollte. Oder PUDOR (1672: 53): In der Mitte unter Nummer 3 ist eine Erzehlende [Sentenz], die man nach der Konjunktion unterscheiden kann, die folgende Einteilung sollte also eigentlich ein bisschen weiter rechts eingerückt werden.
}

16 Vgl. etwa Pudor (1672: 107, 137).

17 Vgl. etwa Pudor (1672: 36, 37). 
keine Fehler gibt. Das System von Pudor ist ausgearbeitet und komplex, es nutzt die ganze Fläche. Es kann zwar im ersten Moment auf den Leser intimidierend wirken, hat sich der Leser jedoch einmal an dieses System gewöhnt, orientiert er sich im Text ohne Probleme, und es lässt sich mit ihm leicht arbeiten.

\section{Eine Parallele in der Logik}

Christian Pudor war nicht der einzige, dessen Bemühungen um eine übersichtliche Textgliederung eine starke Herausforderung für den Buchsetzer mit sich brachte. Eine analoge Vorgehensweise lässt sich in der Logik bei Gottlob Frege beobachten. Die heute übliche Notation $^{18}$, die von Giuseppe Peano und Bernhard Russell stammt, ist auf den ersten Blick stärker linear geprägt. Die Formel wird auf einer Zeile hintereinander gereiht. Frege entwickelte in seiner Begriffsschrift (1879) eine, seiner Meinung nach, anschaulichere Notationsweise. Er versucht die Beziehungen zwischen Prämissen und Konklusionen räumlich darzustellen und unterstützt seine Bemühung mit folgender Argumentation: Nach Frege nutzt Peano die Möglichkeiten und Vorteile der geschriebenen Sprache nicht völlig aus und verzichtet so auf die Übersichtlichkeit der Notation. Eine einzeilige Formel sei schwieriger mit einem Blick zu übersehen und deshalb auch zu verstehen, ihre Gliederung sei nicht so übersichtlich. Für das menschliche Wahrnehmen seien kurze Zeilen, die untereinander stehen, viel bequemer, und sie erlaubten es auch, die Struktur intuitiver zu entziffern. Das einzige Hindernis und somit der Grund für den Verzicht auf die bessere, also zweidimensionale, Darstellungsweise sei die große Anforderung an den Setzer. Das ist für Frege (1896: 378) aber kein ausreichender Grund. Er konstatiert,

„dass die Peano'sche Bezeichnungsweise ohne Zweifel für den Setzer bequemer ist und vielen Fällen auch weniger Raum in Anspruch nimmt als meine eigene, dass diese Vortheile mir aber durch geringere Uebersichtlichkeit und logische Mängel zu theuer erkauft scheinen, wenigstens für die von mir verfolgten Zwecke.“

Es lässt sich darüber streiten, welche der beiden Weisen übersichtlicher oder logischer ist, jedenfalls hat Frege mit diesen Argumenten wenig bewirkt, und sein System hat sich nicht durchgesetzt.

\section{Misserfolg von Zweidimensionalität}

$\mathrm{Zu}$ Beginn dieses Beitrags haben wir betont, dass es ein Vorteil der geschriebenen Sprache ist, die zwei Dimensionen der Fläche ausnützen zu können. Wir haben Pudors Gliederungsmittel vorgestellt, wo die zwei Dimensionen der Fläche reichlich ausgenutzt wer-

18 Die ist die sogenannte Peano-Russell-Notation; sie geht auf die Werke Formulaire de mathématiques (1895-1908) von Giuseppe Peano und Principia mathematica (1910-1913) von Bertrand Russell und Alfred North Whitehead zurück. 
den, und auch den Ansatz von Frege in der logischen Notation. Zugleich haben wir aber gesehen, dass beide diese Systeme nicht erfolgreich waren. Wir können einen Vergleich erstellen:

\begin{tabular}{|l|c|c|}
\hline & Eindimensional & Zweidimensional \\
\hline Textgliederung & Wittgenstein & Pudor \\
\hline Logische Notation & Peano/Russell & Frege \\
\hline Erfolgreich & ja & nein \\
\hline
\end{tabular}

Warum haben sich also die Systeme, die beide Dimensionen stärker ausnutzen, nicht durchgesetzt? Es ist naheliegend, dies auf typographische Schwierigkeiten zurückzuführen. In beiden Fällen musste es für den Setzer kompliziert sein, alle Elemente richtig zu drucken. Bei Pudor mussten die geschweiften Klammern in der richtigen Position sein, sie mussten korrekt bezeichnet werden, und der Text musste an den richtigen Stellen eingerückt werden. Bei Frege mussten die Linien und Stufen passen. Auch heute wären solche Umsetzungen problematisch. Jeder, der schon einmal einen wissenschaftlichen Text verfasst hat, hat wahrscheinlich auch die Nummerierung mithilfe von einer Liste mit mehreren Ebenen erstellt. Das ist in gängigen Texteditoren wie Microsoft Word nicht allzu kompliziert. Es wäre aber wesentlich aufwendiger, den Text alternativ mit geschweiften Klammern zu versehen.

Ein weiterer Nachteil ist der Raumbedarf. Die pudorschen Klammer nehmen Platz an dem linken Rand ein. Dabei wird der Text, nicht nur die Überschriften, eingerückt. Dadurch nimmt der Text mehr Seiten in Anspruch. Bei Frege kann man die Formeln nicht einfach im Rahmen des Textes anführen. Sie müssen gesondert stehen, um übersichtlich zu bleiben.

Der Ertrag dieser ausgearbeiteten Systeme scheint offenbar nicht ausreichend zu sein, um die technischen Schwierigkeiten zu rechtfertigen. Für manche Leser sind sogar diese ausgearbeiteten komplexen Darstellungsweisen gar nicht so übersichtlich und nützlich, wie ihre Autoren meinten, sondern eher verwirrend und unüberschaubar. Dennoch handelt es sich um bemerkenswerte Ansätze, wobei gerade deren Scheitern aus schrifttheoretischer Sicht besonders interessant ist.

\section{Quellen und Literatur}

BÖDIKER, Johann (1690): Grund-Sätze Der Deutschen Sprachen im Reden und Schreiben. Cölln an der Spree.

BRINK, Alfred (2007): Anfertigung wissenschaftlicher Arbeiten. 3. Aufl. München/Wien: Oldenbourg.

FREGE, Gottlob (1879): Begriffsschrift. Eine der arithmetischen nachgebildete Formelsprache des reinen Denkens. Halle: Nebert.

FREGE, Gottlob (1896): "Ueber die Begriffsschrift des Herrn Peano und meine eigene". In: Berichte über die Verhandlungen der königlich sächsischen Gesellschaft der Wissenschaften zu Leipzig. Mathematisch-physische Classe. Bd. 48. Leipzig: Hirzel, S. 361-378. 
GUEINTZ, Christian (1641): Deutscher Sprachlehre Entwurf. Cöthen.

JELLINEK, Max Hermann (1913-1914): Geschichte der neuhochdeutschen Grammatik von den Anfängen bis auf Adelung. 2 Bde. Heidelberg.

MELANCHTHON, Phillip (1573): Grammatica latina. Leipzig.

MOULIN-FANKHÄNEL, Claudine (1997): Bibliographie der deutschen Grammatiken und Ortographielehren. II. Das 17. Jahrhundert. Heidelberg.

PEANO, Giuseppe (1985-1908): Formulaire de mathématiques. 5 Bde. Turin: Bocca frères.

PUDOR, Christian (1672): Der Teutschen Sprache Grundrichtigkeit und Zierlichkeit. Cölln an der Spree.

RAIBLE, Wolfgang (1991): Die Semiotik der Textgestalt. Erscheinungsformen und Folgen eines kulturellen Evolutionsprozesses. Heidelberg: Carl Winter Universitätsverlag.

RINAS, Karsten (2014a): „Zeichensetzung und Syntax: Christian Pudors Interpunktionslehre (1672)“. In: Brünner Beiträge zur Germanistik und Nordistik 28/2014, Nr. 1-2, S. 3-14.

RINAS, Karsten (2014b): "Von der Rhetorik zur Syntax: Die deutsche Interpunktionslehre im Zeitalter der Aufklärung”. In: Sprachwissenschaft 39/2, 2014, S. 115-181.

RUSSELL, Bernhard/ WHITEHEAD, Alfred North (1910-1913): Principia mathematica. 3 Bde. Cambridge: University Press.

SCHOTTELIUS, Georg Justus (1663): Ausführliche Arbeit Von der Teutschen Haubt Sprache. Braunschweig.

STEFFENS, Franz (1909): Lateinische Paläographie. 125 Tafeln in Lichtdruck mit gegenüberstehender Transkription nebst Erläuterungen und einer systematischen Darstellung der Entwicklung der lateinischen Schrift. Trier. http://www.paleography.unifr.ch/steffens_fr/ (25. 2. 2017).

STIELER, Kaspar (1691): Kurze Lehrschrift Von der Hochteutschen Sprachkunst. Als Anhang zu ders. Der teutschen Sprache Stammbaum und Fortwachs. Altdorf.

WEISE, Christian (1691): Curiösen Gedancken von Deutschen Brieffen. Dresden.

WITTGENSTEIN, Ludwig (1922): Tractatus Logico-Philosophicus. London: Kegan Paul, Trench, Trubner \& Co.

Mgr. Jana Adamusová / jana.adamusova@centrum.cz

Univerzita Palackého v Olomouci, Filozofická fakulta, Katedra germanistiky

Kř́žkovského 10, 77180 Olomouc, CZ

doc. Dr. phil. PhDr. Karsten Rinas / K.Rinas@seznam.cz

Univerzita Palackého v Olomouci, Filozofická fakulta, Katedra germanistiky

Kř́ržkovského 10, 77180 Olomouc, CZ 
R DE COMITIIS HABENDIS

Ex IIVIris eni ninc.sunt Item - ex - is - edi

LII DEINCEPS - IN $\cdot$ EO - MVNICIPIO - TIVIRI - ERVNT

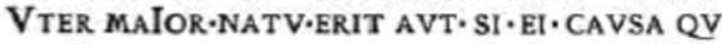

AE INCIDERIT Q M COMITIA HABERE POS

SIT - TVM - ALTER - EX HIS - COMITIA - IIVIR - ITEM

AEDILIBVS - ITEM QYAESTORIBVS - ROGANDIS

SVBrogandis $\cdot \mathrm{H} \cdot \mathrm{L} \cdot \mathrm{HABETO} \cdot \mathrm{VtI}$ TyE EA DIS

TRIBVTIONE- CVRIARVM DEQVA - SVPRA. CON

Prehensvm - est - SvFFragia - Ferri - Debe

BVNT. ITA - PERTABELLAM - FERANTVR FACITO QVIQVE-ITA CREATI - ERVNT - II - ANNVM - VNM AVT - SI I IN AL TERIVS - LOCVM - CREATI - ER VNT RELIQYA P PARTE - EIIVS ANNI - IN EO - HONORE SVNTO-QVEM.SVFFRAGIS-ERVNT-CONSECVTI

R INQYA. CVRIA. InCOLAE SVFFragia FERANT

Qvi.Cvmeve.In eo.mvnicipIo comitia II virIs

LiII Item aEdILIBVS ITEM QVAESTORIBVS ROGAN DIS - HABEBIT EX C CVRIIS - SOR TE.DVCITO VNAM

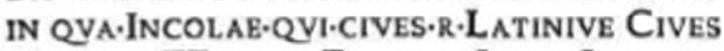
ERVNT SVFFragio.FERANT-EIsQVE.IN EA.CV RIA - SvFFragi - LAtio ESto

R QVORVM - COMITIS - RATIONEM HABE RI OPORTEAT

QVI
COMITIA - HABERE.DEBEBIT-IS - PRIMVM-IIVIR GENVORVM - HOMINVM - DE QYO H - L - CAV TVM CONPREHENSVMQYE - EST - DEINDE - PROXI MO-QYOQYE-TEMPORE-AEDILES-ITEM-QYAESTO RES - EX - EO - GENERE - INGENVORVM - HOMINM DE QVO.H.L.CAVTVM. CONPREHENSVMOYE-EST CREANDO - CVRATO - DVMNE - CVIIVS COMI TIS - RATIONEM - HABEAT - QYI - IVIRATVM - PE TET-ET-QYI MINOR'ANNORVM - XXV ERIT QVI VE. INTRA QVINQYENNIVM - IN - EO - HONORE FVerint Item QVi aediLit ATEM-QVAestVRam VE.PETET- QYI MINOR- QYAM ANNOR XXV ERIT QVIVE - IN - EARVM - QYA- CAVSA-ERIT.PROPTER

Abbildung 1: Ausschnitt aus dem Stadtgesetz von Málaga. In: RAIBLE (1991: 7) 


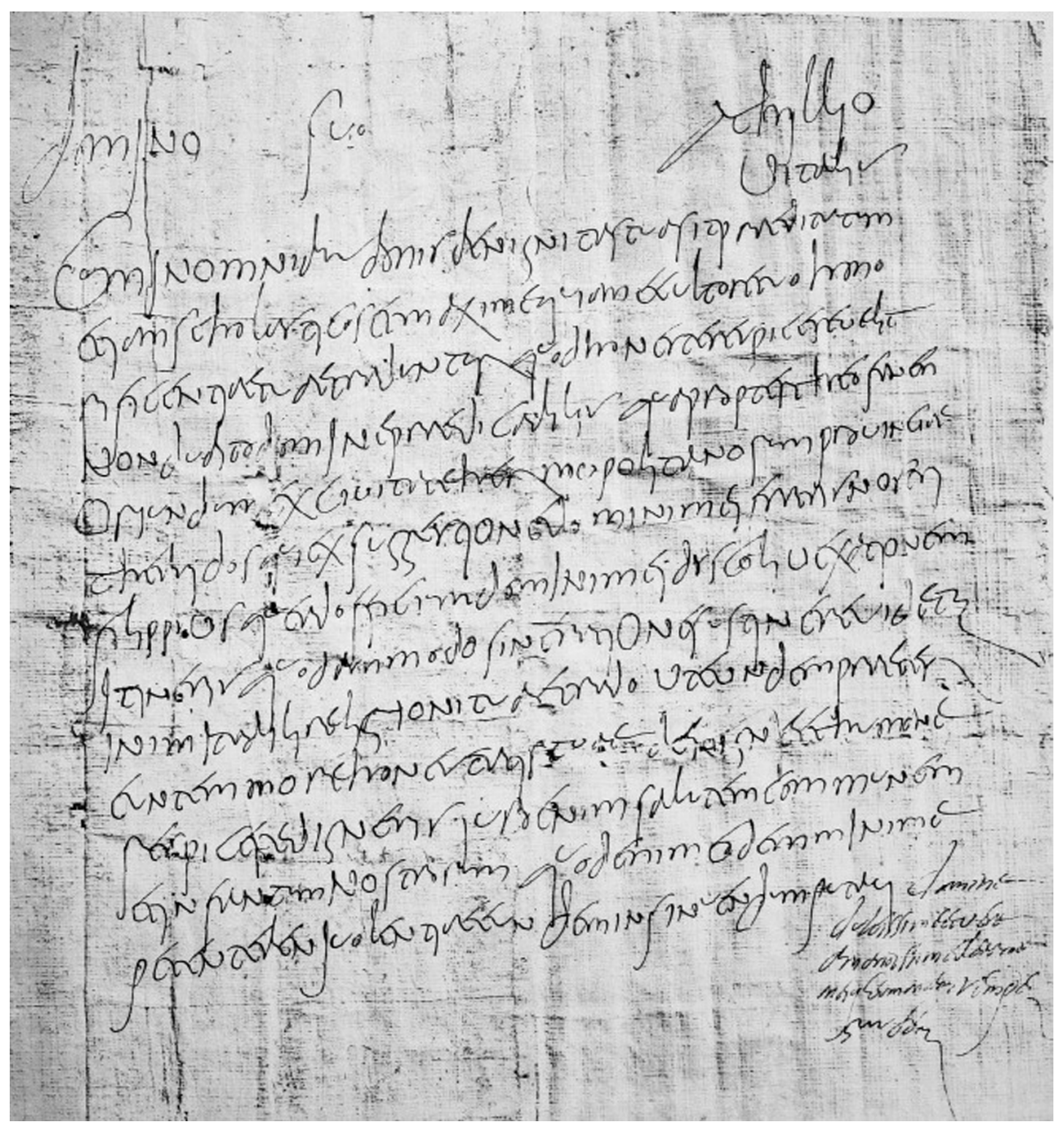

Abbildung 2: Lateinischer Brief aus dem 4. Jahrhundert. In: STEFFENS (1909: 13) 


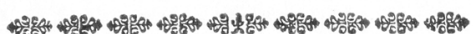

Žurt3er 建ntwutf Seb ganten Trațätebens. B̈egenwärtige Tabellen fellen liirn̨lich fuir

Der Teutfaen Spracte

Sicunorichtigfsit.

\section{FUND A MEN T A. p. $r$.}

Dabel) zu beobachten

(1) Die 23dh/taben/und bero

(1 3abl. p.1.

$\{2$ 2rbtheilnng. p. 1.

3 2(uffiurect)ung. p. 2.

14 Sobreibung. p.7.

2 Die Sylben. P.14

, Die Dodrter. P. 15.

1 Ing gemein/nadh ifrer

1 2rbtheilung/fis werosnaber abgetteilet nato

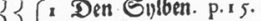

$\{\{2$ Der ableitung. p.is.

I 13 Der Bctboppelung. p.x6.

(4 Der Zubluandelung. p. 17 .

2 2(uffiprectiang. p. 17

3 Sdbreibunt P. 18.

2 Infonderbeit wito betractetet)

(1 Das (S) efthlect)tsoluott/ felbiges if

S Sin Zeutunendes. p.2 3 .

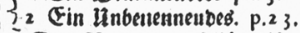

2 Das Tennmortl felbiges if

( Stibitandig

I Eigell. p.25.

$\{2$ Gemein. p. 27.

2 Benftàndig.p. 30 . Daben ju batracbten beffen

(1 Enberung. (Morio.) p.r3.

$\{$ 3ergrefferung. (Comparatio.) p. 31 .

32 2tonulubchung. (Declinatio.) p. 32.

3 Das Dothentwodt. p. 32.

4 Ing Zeitwort. P. 33 .

5 Das 5 Mittclivort. p.4

6 Dab 3utwort. p.42.

7 Dak Borluort. P.43.

8 Das sfigewort. p. 43 .

4 Die Redensarten (Phrafes.) p. 44.

5 Die Sprucbreden. (Sententix.) p. 5.

6 Die Sabluffredent. (Periodi.) p.ss.

(7) Eiue ganne voltemmente Teut/dye Krese. (Oratio.) p.r6

\section{II}

Bierlidffeit.

ORNAMENTA. p.s9.

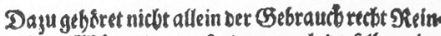

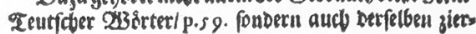
ticlve

1 Derferzung. p. 64.

2 Dermed jelung. p.72.

$\{3$ 任rmeiterung. p. 85 .

4 Zurammenzichung. p.97.

5 und cutliclvoiereckmapisige Dersmadbung.p.ro\%.

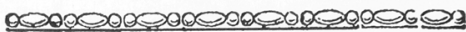

Abbildung 3: Inhaltverzeichnis. In: PUDOR (1672) 


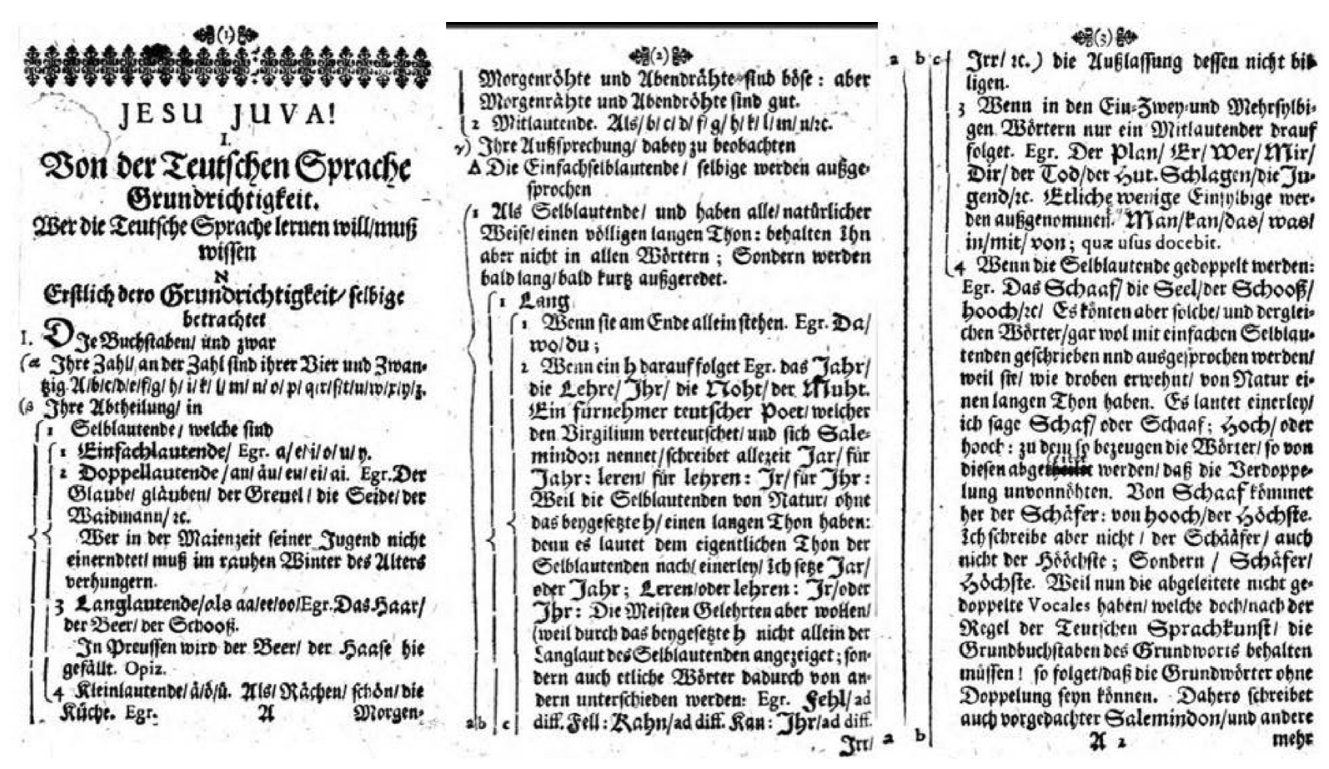

Abbildung 4: Die ersten drei Seiten aus PUDOR (1672).

heute übliche Notation:

$$
\mathrm{P} \supset \mathrm{Q}
$$$$
\mathrm{P} \supset(\mathrm{Q} \supset \mathrm{R})
$$

$$
\neg(\mathrm{P} \supset \neg \mathrm{Q})
$$

FREGEs Notation:

$$
\tau_{P}^{Q}
$$

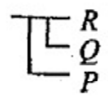

TP $P$

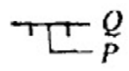

Abbildung 5: Beispiele für die Notation in FREGE (1879) 